\title{
Drug-resistance reversal in colorectal cancer cells by destruction of flotillins, the key lipid rafts proteins
}

\author{
D. M. YE $E^{1,2, *}$, S. C. YE $E^{1, *}$, S. Q. YU ${ }^{1,2}$, F. F. SHU ${ }^{1,2}$, S. S. XU ${ }^{1,2}$, Q. Q. CHEN ${ }^{1,2}$, Y. L. WANG ${ }^{2}$, Z. T. TANG ${ }^{2}$, C. PAN ${ }^{1,2, *}$ \\ ${ }^{1}$ School of Medicine, Xiamen University, Xiamen, Fujian, China; ${ }^{2}$ Department of Pathology, Zhongshan Hospital affiliated to Xiamen University, \\ Xiamen, Fujian, China
}

*Correspondence: panchao@xmu.edu.cn

${ }^{*}$ Contributed equally to this work.

Received August 20, 2018 / Accepted January 30, 2019

\begin{abstract}
Multidrug resistance (MDR) of tumor cells attenuates the efficacy of anticancer drugs and has become the main reason for chemotherapy failure. It is indispensable to establish an effective way to reverse multidrug resistance. Our previous work has shown that downregulation of the ERK/MAPK signaling pathway activity can reverse the drug resistance of resistant cells. Furthermore, the effect of signal transduction is strongly associated with lipid rafts. The drug resistance is reversed successfully after lipid rafts are destroyed by heptakis(2,6-di-O-methyl)- $\beta$-cyclodextrin $(\mathrm{M} \beta \mathrm{CD})$. However, reversal of the drug resistance is not associated with downregulation of ERK1/2 expression. Cell membrane permeability may increase when lipid rafts are destroyed by $\mathrm{M} \beta \mathrm{CD}$, causing the reversal of drug resistance due to an increased drug accumulation in cytoplasm. To minimize the influence of $\mathrm{M} \beta \mathrm{CD}$ on the cell membrane structure, we selected flotillin, a marker protein of lipid rafts, as the target molecule to further investigate the mechanism of changes in drug resistance after destruction of lipid rafts. The effect of flotillin on the reversal of drug resistance was examined using RNA interference (RNAi) in a retroviral system in human drug resistant strains of colorectal cancer cell line HCT-15. The results demonstrate that flotillin-1 downregulation by RNAi (Flot1-RNAi) reduced the drug resistance, caused cell cycle arrest and decreased the expression of ERK1/2; however, apoptosis was not significantly affected. Knockdown of flotillin-2 by RNAi (Flot2-RNAi) had effects similar to those of Flot1-RNAi except that the effects on ERK1/2 expression and apoptosis were different. Screening of multiple pathways indicated that the PI3K/Akt signaling pathway was closely related. This experiment demonstrates an association between PI3K and drug resistance through the activation of PI3K and suggests that PI3K may play a key role during the development of resistance in CRC. The results reveal that the levels of IRS-1 and PI3K proteins in the Flot1RNAi and Flot2-RNAi groups were significantly downregulated. Knockdown of flotillins by RNAi reduced the resistance of HCT-15/ADM cells; and the results on Akt pathway indicate a decrease in resistance after lipid raft destruction. These data confirm that knockdown of flotillin reduces the resistance of HCT-15/ADM cells and the mechanism may be relevant to the PI3K/Akt pathway. Additionally, flotillin may be used as a potential target for chemotherapy in the treatment of colorectal cancer.
\end{abstract}

Key words: colorectal cancer, CRC, flotillin, RNAi, PI3K, ERK1/2

Colorectal cancer (CRC) is one of the common malignant carcinomas in the world [1]. Currently, excision combined with chemotherapy remains the most significant approach of the CRC treatment. Unfortunately, this approach frequently fails because of drug resistance [2]. Thus, it is important to identify the mechanisms involved in drug resistance, to identify potential targets for diagnostic or therapeutic strategies.

Membrane molecules span across the cell membrane and transmit signals from the external environment to the inside of cells and their distribution on the cell surface is non-uniform. Membrane lipid rafts are the specialized domains in cell membranes that can be selectively enriched in certain proteins and bind the proteins, functioning as the physical platforms for protein-protein interactions [3-6]. Lipid rafts play important roles in a number of biological processes including transmembrane signal transduction, endocytosis, lipid and protein sorting [4, 7-11]. In recent years, several scientists suggested that lipid rafts are associated with drug resistance [12-14].

Flotillin-1 and -2, also known as Reggie-2 and -1, respectively, are two highly conserved homologous isoforms of 
the lipid raft-associated proteins originally described in the retinal ganglion cells during axon regeneration $[15,16]$. Flotillins participate in various cellular processes, such as signal transduction, endocytosis, $\mathrm{T}$ cell activation and interaction with the cytoskeleton [17-20]; however, it is unclear whether these mechanisms function in the resistant CRC cells.

The ERK1/2 cascade is the most widely studied module of the mitogen-activated protein kinase (MAPK) signaling pathways $[21,22]$. Our previous study demonstrated that ERK1/2 activity is upregulated in resistant cells [23]. A number of studies indicated that the ERK1/2 signaling pathway is associated with drug resistance. Increased resistance of MCF7 tumor cells to doxorubicin is linked to activation of ERK1/2 signaling pathway and increased expression of MDR and p-gp [24]. After PD98059 treatment, the sensitivity to MDR was significantly increased and the drug reversed the resistance in human lymphoma cells [25].

The phosphoinositide 3-kinase (PI3K)/Akt signaling pathway may be closely associated with cell growth, survival and apoptosis [26]. PI3K is mainly activated via three pathways: 1) it directly binds to the phosphorylated receptor tyrosine kinase (RTK); 2) insulin receptors bind to the insulin receptor substrates (IRS-1) [27]; 3) it binds to the GTP-bound form of Ras. PI3K is activated by various growth factors and cytokines to produce phosphatidyleinositol $(3,4,5)$-triphosphate, $\operatorname{Ptd} \operatorname{Ins}(3,4,5) \mathrm{P} 3$, which in turn activates a number of important downstream proteins [28-31]. Akt, a serine-threonine protein kinase, is a central player in tumor development [32] and the characteristic downstream target of PI3K. The activity of PI3K/Akt family has been involved in the regulation of cell apoptosis, cell cycle and tumor development [33]. Recent evidence indicates that phosphorylation of Akt induces drug resistance in several tumors, such as ovarian cancer [34], breast cancer [35] and hepatocellular carcinoma [36]. The aim of the present study was to knockdown flotillin expression to investigate whether flotillins influence the multidrug resistance of resistant CRC cells.

\section{Materials and methods}

Cell culture. Human colorectal cancer cell line HCT-15 was obtained from the Institute of Cell Biology, Chinese Academy of Sciences. The cells were pulsed with highconcentrations of doxorubicin (ADM) for an extended period of time and the half-inhibitory concentration (IC50) was calculated by assessing the sensitivity to various concentrations of ADM by the Cell Titer 96R AQueous One Solution (MTS) (Promega Biotech Co., USA). Human embryonic kidney cell line 293FT was obtained from Central Laboratory of Zhongshan Hospital affiliated with Xiamen University. HCT-15 and 293FT cells were cultured in RPMI 1640 (Gibco; Thermo Fisher Scientific, USA) containing 10\% fetal bovine serum (FBS; HyClone, USA), $100 \mathrm{U} / \mathrm{ml}$ penicillin and $100 \mu \mathrm{g} / \mathrm{ml}$ streptomycin (Invitrogen; USA).
Lentiviral packaging vector. The lentiviral backbone plasmid PGLV3 was provided by Shanghai GenePharma Co. The PMD and PSPA plasmids were provided by Cai Bo, a laboratory affiliated with Zhongshan Hospital of Xiamen University. PGLV3 is capable of expressing the green fluorescent protein (GFP) reporter through the CMV promoter. Additionally, PGLV3 includes the H1 RNA polymerase III promoter of the shRNA insert that initiates the expression of siRNA providing for continuous interference.

Construction of lentiviral vectors. The target genes, flotillin-1 (NM_005803. 3) and flotillin-2 (NM_004475. 2) were retrieved from the NCBI (National Center for Biotechnology Information) website, and a Blast search was performed to determine the validity of the sequence. Finally, we selected a shRNA sequence for each isoform: 833-851 of flotillin-1 (Flot1-RNAi) and 604-622 of flotillin-2 (Flot2RNAi). Scrambled shRNA sequence (5'- GATCCGTTCTCCGAACGTGTCACGTTTCAAGAGAACGTGACACGTTCGGAGAACTTTTTTG-3') was used as a control (Con). The lentiviral vector PGLV3 was cut with two enzymes, BamHI and EcoRI (Thermo; Thermo Fisher Scientific) and ligated by T4 ligase (Thermo; Thermo Fisher Scientific), the concatenated product was transformed into E. coli DH5a (Tiangen Biotech (Co), and sequenced to extract the recombinant interference plasmid.

The shRNA-containing lentiviruses (Con, Flot1-RNAi and Flot2-RNAi) were generated by co-transfecting the 293FT cells by Turbofect with the transfer and packaging vectors: shRNA-PGLV3, PMD and PSPA. After $48 \mathrm{~h}$ the supernatants containing lentiviruses including Con, Flot1-RNAi and Flot2-RNAi were harvested.

Infection with lentivirus. HCT- 15 cells in the logarithmic growth phase were seeded in six-well plates and cultured overnight; then, the cells were infected with recombinant lentiviruses. After $24 \mathrm{~h}$, the percentage of GFP-positive cells was estimated using a fluorescence microscope to determine the infection efficiency. After $96 \mathrm{~h}$ post infection, $2 \mu \mathrm{g} / \mathrm{ml}$ puromycin was added to select the stable transformants.

Western blot analysis. The infected cells were scraped on ice and collected by centrifugation. Lysis buffer was freshly prepared and added to the infected cells and incubated for 30 min. Cell extracts were prepared by centrifugation and the protein concentrations were determined by using a protein analysis kit (BCA). Equal amount of protein $(30 \mu \mathrm{g})$ was boiled for $10 \mathrm{~min}$ before SDS-PAGE separation. Separated proteins were transferred to polyvinylidenedifluoride (PVDF) membranes (Millipore, Bedford, USA) at $300 \mathrm{~mA}$ for $1 \mathrm{~h}$ and the membranes were blocked in TBST buffer with 5\% nonfat milk. Anti-flotillin-1, anti-flotillin-2 (Abcam, USA), anti-ERK1/2, anti-p-ERK1/2 (Cell Signaling Technology, USA), anti-IRS-1, anti-PI3K (Wanleibio, China) and antiGAPDH (Abways, XiaMen YiRu Biotechnology, China) were diluted 1:10000, 1:4000, 1:1000, 1:1000, 1:300, 1:1000 and $1: 7000$, respectively and incubated at $4^{\circ} \mathrm{C}$ for $12 \mathrm{~h}$. Secondary antibodies (Jackson ImmunoResearch Laboratories, Inc., 
West Grove, PA, USA) were incubated for $1 \mathrm{~h}$ at room temperature on a shaker and the membrane was washed three times for $5 \mathrm{~min}$ with TBST. Protein bands were visualized by using WesternBright ECL (WesternBright ECL; USA).

Drug susceptibility test of ADM in drug resistant cells. According to the Cell Titer 96 AQueous One Solution Cell effort assay kit instructions, the cells were plated at density of 3000 cells per well in 96-well microtiter plates and peripheral wells were filled with PBS to reduce the edge effect. Plates were incubated overnight at $37^{\circ} \mathrm{C}$ in a humidified incubator containing $5 \% \mathrm{CO}_{2}$; then the cells were carefully washed three times with PBS. To investigate the drug resistance of resistant cells, the parental cells and resistant cells were exposed to various concentrations of $\mathrm{ADM}(0,0.25$, $1,4,16$ or $64 \mu \mathrm{g} / \mathrm{ml})$. Each drug concentration was tested in 5 replicates. After conventional culture for $24 \mathrm{~h}$, the cells were washed with PBS and $100 \mu$ fresh complete culture medium was added; then, $20 \mu \mathrm{l}$ MTS solution was added to each well at a ratio of 5:1 in the dark and incubated $2 \mathrm{~h}$. The OD of each well was determined at $490 \mathrm{~nm}$. If the differ-

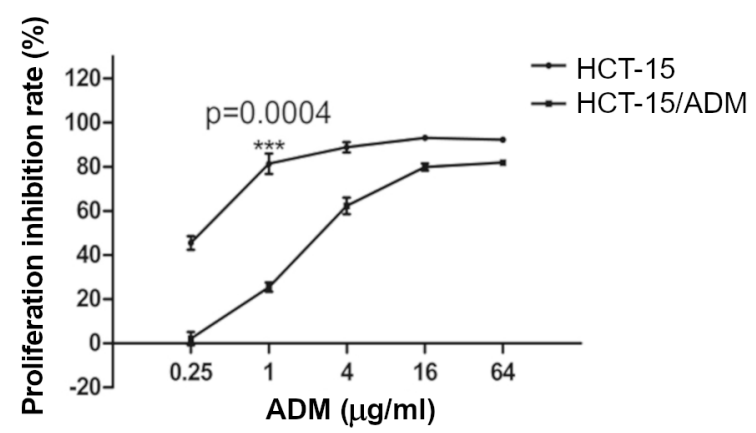

Figure 1. Increased drug resistance of HCT-15/ADM cells to ADM compared to HCT-15 cells. The HCT-15 and HCT-15/ADM cells were incubated with $0,0.25,1,4,16$ or $64 \mu \mathrm{g} / \mathrm{ml} \mathrm{ADM}$ for $24 \mathrm{~h}$. At the end of incubation, the cell survival rates were determined by the Cell Titer 96 AQueous One Solution Cell effort assay in three independent experiments performed in five replicates; the proliferation inhibition rate was calculated. Results are reported as the mean \pm standard deviation.

Table 1. ADM IC50 in HCT-15 and HCT-15/ADM cells.

\begin{tabular}{lcc}
\hline Cell line & IC50 (ADM $\mu \mathrm{g} / \mathbf{m l})$ & Resistance Index $(\mathrm{RI})$ \\
\hline HCT-15 & $0.2 \pm 0.013$ & \\
HCT-15/ADM & $3.516 \pm 0.26$ & $17.58^{\star * *}$ \\
\hline
\end{tabular}

${ }^{* * *} \mathrm{p}<0.001$, IC50 inhibitory concentration; ADM, adriamycin.

Table 2. ADM IC50 in CRC resistant cells treated with Con, Flot1-RNAi and Flot2-RNAi.

\begin{tabular}{lcc}
\hline Cell line & IC50 $(\mathrm{ADM} \mu \mathrm{g} / \mathrm{ml})$ & Reverse fold \\
\hline HCT-15/ADM + Con & $3.456 \pm 0.215$ & \\
HCT-15/ADM + Flot1-RNAi & $2.507 \pm 0.026$ & $1.38^{* *}$ \\
HCT-15/ADM + Flot2-RNAi & $1.57 \pm 0.073$ & $2.2^{* *}$ \\
\hline
\end{tabular}

${ }^{* *} \mathrm{p}<0.01 ;{ }^{* * *} \mathrm{p}<0.001$. ence between two wells was greater than $5 \%$, the data were considered as invalid. The cell proliferation inhibition rate was calculated by the following equation: cell proliferation inhibition rate $=1-$ (experimental group OD - blank control group OD) / (negative control group OD - blank control group OD). The half-maximal inhibitory concentration of the drug (IC50) was estimated by the SPSS software.

Cell apoptosis analysis. Cells in logarithmic growth phase were plated in 6 -well plates at $1 \times 10^{6}$ cells per well. Cells treated with $1 \mu \mathrm{g} / \mathrm{ml}$ ADM for $24 \mathrm{~h}$, digested by trypsin without EDTA and collected. The cells were washed twice with cold PBS and then resuspended in 1x Annexin $\mathrm{V}$ binding solution (Dojindo Molecular Technologies, Shanghai, China) at a concentration of $1 \times 10^{6}$ cells $/ \mathrm{ml}$; then, $5 \mu \mathrm{l}$ Annexin V-633 conjugate and $5 \mu \mathrm{l}$ propidium iodide (PI) solution were added to the cell suspension in the dark. Samples were incubated for $15 \mathrm{~min}$ at $37^{\circ} \mathrm{C}$. $1 \times$ Annexin V binding solution was added $(400 \mu \mathrm{l})$ and samples were assayed by flow cytometry (Beckman Coulter, USA) within $1 \mathrm{~h}$.

Cell cycle analysis. The infected Con, Flot1-RNAi and Flot2-RNAi cells were plated into 6 well plates at a density of $10^{6}$ cells/well and cultured for $24 \mathrm{~h}$. The cells were harvested by trypsinization, fixed with $95 \%$ ethanol overnight at $4{ }^{\circ} \mathrm{C}$ and resuspended in PI. DNA content was detected by a flow cytometer. The relative proportion of cells in the cell cycle phase fraction was determined according to flow cytometry data.

Statistical analysis. All experiments were performed in triplicate and data are shown as mean \pm standard deviation where applicable. Statistical analysis was performed by using the GraphPad Prism 5 and SPSS version 17. 0 software. Student's t-test, ANOVA and post hoc Student-NewmanKeuls variance test were used for the statistical analyses; and $\mathrm{p}<0.05$ was considered statistically significant.

\section{Results}

HCT-15/ADM cells exhibit stable drug resistance. The ADM IC50 values in the HCT-15 and HCT-15/ADM cells were $0.2 \pm 0.013 \mu \mathrm{g} / \mathrm{ml}$ and $3.516 \pm 0.26 \mu \mathrm{g} / \mathrm{ml}$, respectively and the resistance index of HCT-15/ADM cells was 17.58 . The results are shown in Figure 1 and Table 1. The data indicate that the difference in IC50 values between parental cells and resistant cells is significant $(\mathrm{p}<0.001)$, indicating that HCT-15 cells exhibited stable chemoresistance.

Knockdown of flotillins in resistant colorectal cancer cells by shRNA. To knockdown flotillin expression, a lentiviral vector system containing shRNAs was directed against the flotillin gene. Additionally, GFP was incorporated as a reporter gene. After a single exposure of HCT-15/ADM cells to the constructed lentivirus, a high percentage (more than 90\%) of the infected cell expressed GFP $96 \mathrm{~h}$ after infection (Figure 2A). These results indicated that the highly efficient and stable lentiviral vector targeting the flotillins gene was successfully constructed. 
A
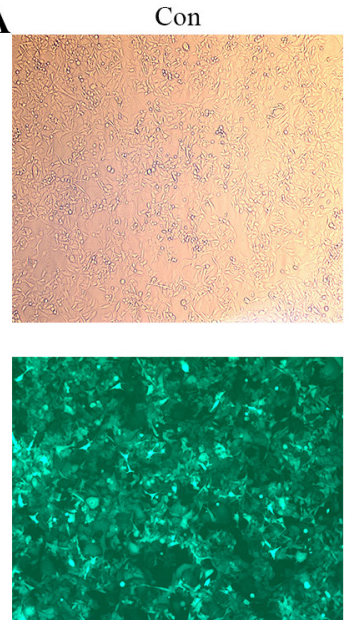

Flotl-RNAi
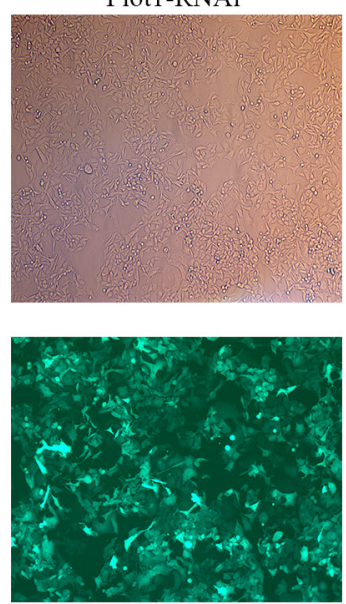

Flot2-RNAi
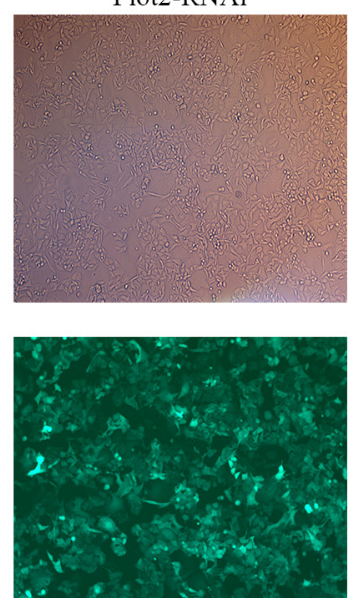

B

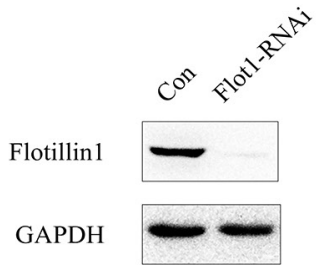

Flotillin2

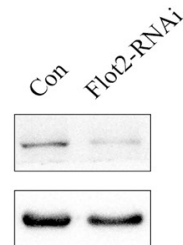

Figure 2. Knockdown of flotillin-1 and flotillin-2 by shRNA in HCT-15/ADM cells. Fluorescence microscopy assay of lentiviral infection efficiency in HCT-15/ADM (A) cells (magnification 40×). Western blot showing a decrease in flotillin-1 and flotillin-2 protein levels in HCT-15/ADM (B) cells by Flot1-RNAi and Flot2-RNAi. Con: cells infected with non-silencing shRNA; Flot1-RNAi: cells infected with flotillin-1 shRNA; Flot2-RNAi: cells infected with flotillin-2 shRNA.
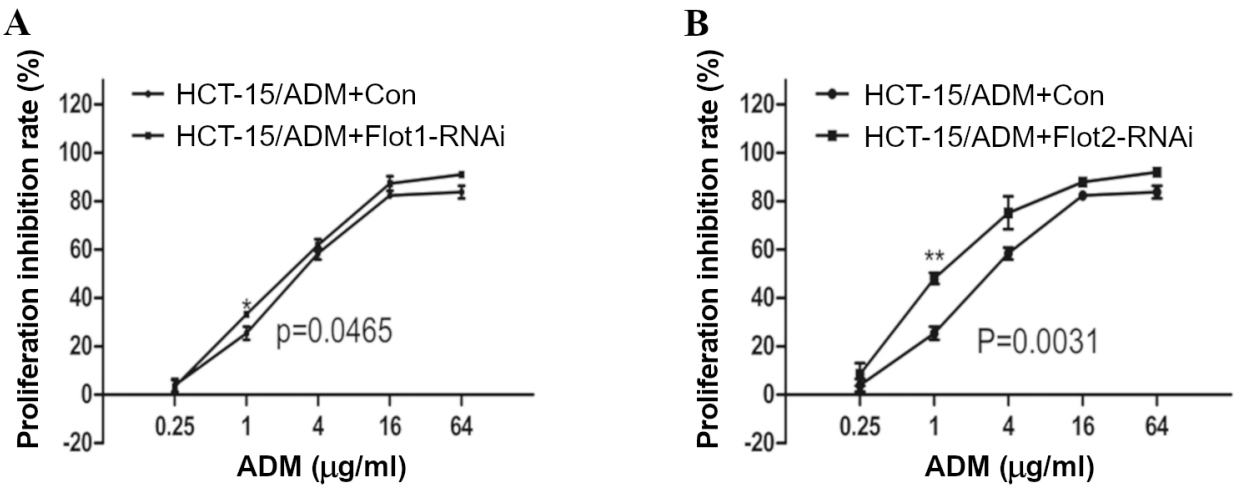

Figure 3. After flotillin knockdown, drug resistance was reduced. A) HCT-15 + Con and HCT-15 + Flot1-RNAi cells were incubated with 0, 0.25, 1, 4, 16 or $64 \mu \mathrm{g} / \mathrm{ml}$ ADM for $24 \mathrm{~h}$. B) HCT-15 + Con and HCT-15 + Flot2-RNAi cells were also incubated with 0, 0.25, 1, 4, 16 or $64 \mu \mathrm{g} / \mathrm{ml} \mathrm{ADM} \mathrm{for} 24 \mathrm{~h}$. At the end of incubation, the cell survival rates were determined by MTS in three independent experiments performed in five replicates; the proliferation inhibition rate was calculated. Results are reported as the mean \pm standard deviation.

shRNA downregulated flotillin expression in resistant colorectal cancer cells. The effect of the flotillin-specific RNAi-expressing lentivirus on the expression of flotillins was examined by western blot analysis in HCT-15/ADM cells. As shown in Figure 2B, the protein levels of flotillins were dramatically reduced in Flot1-RNAi and Flot2-RNAi groups. These data demonstrated that the expression of flotillins was efficiently downregulated by RNAi in resistant CRC cells.

Knockdown of flotillin-1 or flotillin-2 proteins can reverse drug resistance in the drug resistant cells. After the knockdown of flotillin-1 and flotillin-2 in HCT-15/ADM cells, cell sensitivity to ADM increased, IC50 decreased, cell resistance decreased and drug resistance was reversed. Flot1RNAi and Flot2-RNAi cells were treated with repeated doses of $\operatorname{ADM}(0,0.25,1,4,16$ or $64 \mu \mathrm{g} / \mathrm{ml})$; ADM decreased the sensitivity of the cells to ADM and the inhibition of proliferation rate, compared with Con cells (Figure 3). The IC50 of each group of cells was calculated by SPSS. The IC50 values of each group of the cells in HCT-15/ADM cell lines were: Con 3.456 \pm 0.215 ; Flot1-shRNAi 2.507 \pm 0.026 ; and Flot2-shRNAi $1.57 \pm 0.073$; the drug reversal index values were 1.38 and 2.2, respectively (Table 2 ).

Knockdown of flotillin-2 protein promoted apoptosis and knockdown of flotillin-1 had no significant influence. To confirm the effect of flotillins on cell apoptosis, Annexin V-633 double staining of HCT-15/ADM cells was used after lentiviral infection. Flotillin-2 knockdown increased the number of apoptotic cells (early apoptosis and late apoptosis) by 2.1 -fold compared with that in control (Figure 4) and knockdown of flotillin-1 had no significant effect. Hence, 
the data indicate that flotillin-2 inhibition induced a strong proapoptotic effect in resistant CRC cells.

Knockdown of the flotillin protein significantly increased cell cycle arrest. To evaluate the effect of downregulation of flotillins on cell cycle distribution, the PI staining analysis was performed by using a flow cytometer (Figure 5A). As shown in Figure 5B, percentage of cells in the G2/M phase was dramatically decreased in the Flot-RNAi group compared to that in the Con group. These data indicate that downregulation of flotillin expression can induce significant cell cycle arrest.

Expression of flotillin protein was upregulated in the drug resistant strains and flotillin knockdown the expression of PI3K, IRS-1 and ERK1/2 proteins in resistant colorectal cancer cells. The expression of flotillin-1 and flotillin-2 in parental cells and resistant cells was detected by western blot. The results showed that expression levels were significantly higher than those in the parental colorectal cancer cell lines (Figures 6A-B). Then, the expressions levels of PI3K, IRS-1 and ERK1/2 in HCT-15/ADM RNAi cells were detected by western blotting. The results showed that expression was downregulated compared with that in the control group (Figures 6C-D). Knockdown of flotillin-1 reduced the expression levels of phosphorylated ERK1/2 in resistant CRC cells, compared with that in the control group (Con group); however, knockdown of flotillin-2 induced upregulation (Figures 6E-F).

\section{Discussion}

Multidrug resistance of tumor cells remains the main obstacle of cancer chemotherapy despite numerous advances in the treatment of colorectal cancer. Therefore, reversing multidrug resistance is a key factor in tumor treatment. Multiple signal pathways regulate cellular drug resistance. Modulation of cellular drug resistance and related signaling pathways may be a novel therapeutic target in resistant CRC cells.

Our previous studies have demonstrated that downregulation of the ERK/MAPK signaling pathway activity could reverse the drug resistance of resistant cells [37] and that the signal transduction mainly involved lipid rafts $[9,10]$.
A Con

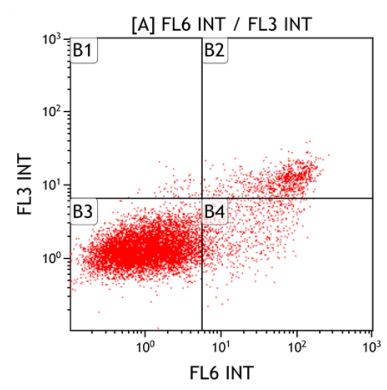

Flot1-RNAi

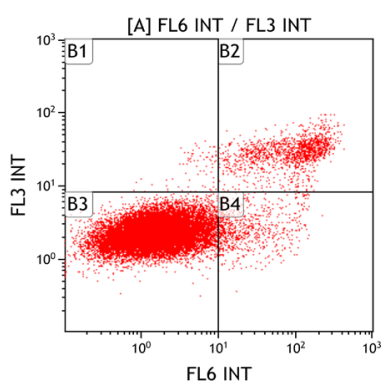

Flot2-RNAi

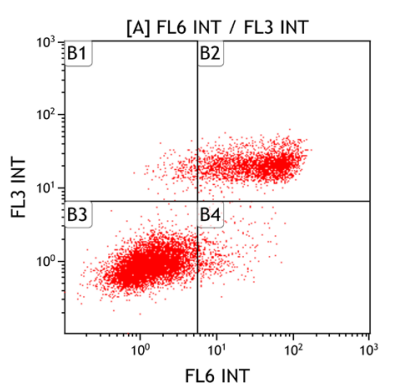

B

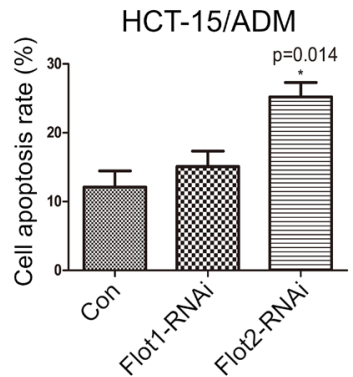

Figure 4. Knockdown of flotillin-2 protein promoted apoptosis and knockdown of flotillin-1 had no significant influence. A) The apoptosis of HCT15/ADM + Con, HCT-15/ADM + Flot1-RNAi and HCT-15/ADM + Flot2-RNAi cells was induced by $1 \mathrm{mg} / \mathrm{l}$ ADM for $24 \mathrm{~h}$. B) Columns represent the means of the average percentage of apoptosis $(\mathrm{B} 2+\mathrm{B} 4)$ in each group of three replicate experiments; error bars indicate standard deviation $(\mathrm{x}$ axis represents the APC-A; $y$ axis represents the PE-A).

A

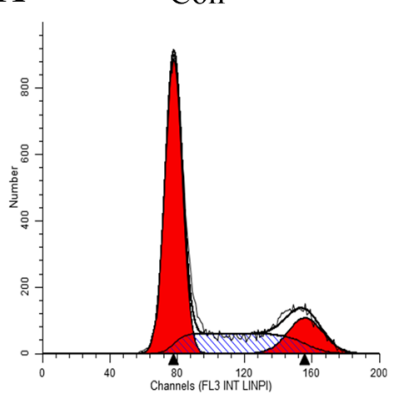

Flot1-RNAi

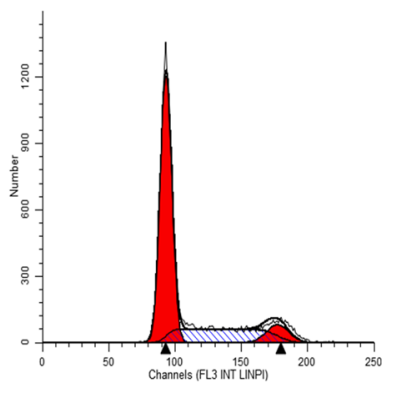

Flot2-RNAi

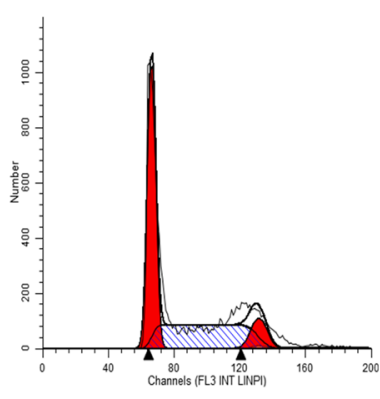

B

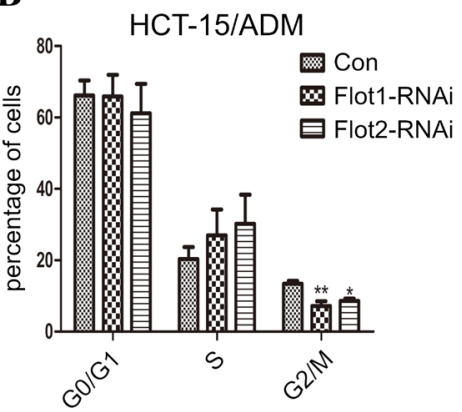

Figure 5. Effect of flotillin silencing on cell cycle progression in HCT-15/ADM cells. A) The changes in cell cycle distribution in HCT-15/ADM (Con, Flot1-RNAi and Flot2-RNAi) cells. B) Columns represent the means of the average percentage of cells in each group of three replicate experiments; bars indicate standard deviation. 

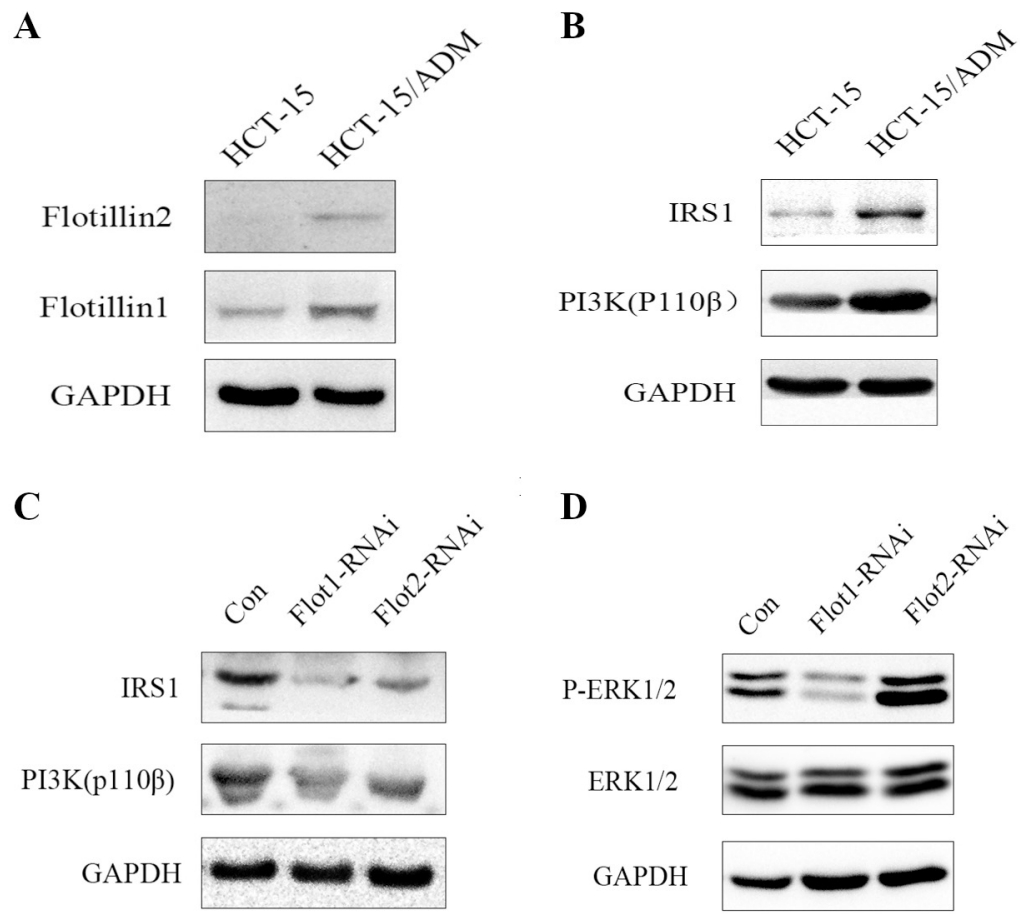

Figure 6. Expression levels of flotillin, PI3K and IRS1 were increased in HCT-15/ADM cells and the expression levels of PI3K, IRS-1 and ERK1/2 proteins in infected cells. A) Flotillin-1 and flotillin-2 protein expression in HCT-15 and HCT-15/ADM cells. B) PI3K and IRS1 protein expression in HCT15 and HCT-15/ADM cells. C) The expression of PI3K and IRS-1 in the knockdown cells. D) The expression levels of total ERK1/2 and phosphorylated ERK1/2 in the knockdown cells (all data were compared with GAPDH as a reference).

Although the resistance of drug resistant cells was decreased after the lipid rafts were destroyed, the ERK1/2 signaling pathway was abnormally upregulated. Based on the results, two hypotheses were proposed; 1) the alteration in drug resistance is related to lipid rafts destruction by $M \beta C D$ accompanied by severe membrane damage leading to increased inward and outward permeability [13]; 2) there may be additional important mechanisms that may have an effect on the colorectal cancer resistance. In this study, the effect of permeability was bypassed by the use of lentivirus-mediated RNAi to knockdown flotillin expression and to investigate the role of flotillins, their impact on the ERK1/2 signaling pathway and to determine whether flotillin can influence the multidrug resistance of resistant CRC cells.

Expression of flotillins in the drug resistant strains was significantly higher than in the parental strains of colorectal cancer cell lines. To determine the role of flotillin in drug resistance and the levels of the ERK1/2 signaling pathway, flotillin-1 and flotillin-2 expression was silenced in resistant CRC cell lines HCT-15 by using lentivirus-mediated RNAi. Our results revealed that the drug resistance was reversed. The drug RI values after knockdown of flotillin-1 and flotillin-2 were 1.38 and 2.2, respectively; the knockdown of flotillin-1 downregulated the pERK1/2 levels without affecting the levels of total ERK1/2; however, knockdown of flotillin-2 induced upregulation of the expression of pERK1/2 without affecting total ERK1/2. A similar phenomenon was observed in the case of flotillin-2 knockdown and lipid raft destruction. Additional important mechanisms may impact the resistance of colorectal cancer; however, permeability cannot be ruled out.

Abnormal changes in the ERK1/2 signaling pathway may be caused by a negative feedback through other signaling pathways. The screening of multiple pathways demonstrated that the PI3K/Akt signaling pathway was closely related according to one of our unpublished studies. We conducted this study of the activation mode of PI3K to prove that the PI3K/Akt signaling pathway is indeed functional. The expression levels of IRS- 1 and PI3K were examined by western blot. The IRS-1 and PI3K levels in the Flot1-RNAi and Flot2-RNAi groups were dramatically downregulated compared with those in the Con. Therefore, the changes in the drug resistance of resistant CRC cells after lipid raft destruction may be related to increased apoptosis and cell cycle arrest after suppression of the PI3K/Akt signaling pathway. Thus, we suggest that the ERK1/2 signaling pathway is one of the therapeutic targets for drug resistance; however, additional major pathways may impact resistance after lipid raft destruction, e.g., the PI3K/Akt signaling pathway. Therefore, future studies of these types of biomolecular interactions are warranted.

In summary, we demonstrated that knockdown of flotillin, the markers of lipid raft, effectively inhibits drug resistance of 
resistant CRC cells. The mechanism of flotillin action may be relevant to the PI3K/AKT signaling pathway. Understanding the precise role played by flotillin in resistant CRC cells will increase our knowledge of the biology of resistant CRC cells and may enable development of a novel therapeutic strategy via suppression of flotillin.

\section{References}

[1] BRODY H. Colorectal cancer. Nature 2015; 521: S1. https:// doi.org/10.1038/521S1a

[2] WU N, FESLER A, LIU H, JU J. Development of novel miR129 mimics with enhanced efficacy to eliminate chemoresistant colon cancer stem cells. Oncotarget 2018; 9: 8887-8897. https://doi.org/10.18632/oncotarget.22322

[3] GAJATE C, MOLLINEDO F. Lipid Rafts, Endoplasmic Reticulum and Mitochondria in the Antitumor Action of the Alkylphospholipid Analog Edelfosine. Anticancer Agents Med Chem 2014; 14: 509-527. https://doi.org/10.2174/1871 520614666140309222259

[4] HANZAL-BAYER MF, HANCOCK JF. Lipid rafts and membrane traffic. FEBS Lett 2007; 581: 2098-2104. https:// doi.org/10.1016/j.febslet.2007.03.019

[5] DAQUINAG A, FADRI M, JUNG SY, QIN J, KUNZ J. The yeast $\mathrm{PH}$ domain proteins $\mathrm{Slm} 1$ and $\operatorname{SIm} 2$ are targets of sphingolipid signaling during the response to heat stress. Mol Cell Biol 2007; 27: 633-650. https://doi.org/10.1128/ MCB.00461-06

[6] VAN MEER G, SIMONS K. Lipid polarity and sorting in epithelial cells. J Cell Biochem 1988; 36: 51-58. https://doi. org/10.1002/jcb.240360106

[7] LINGWOOD D, SIMONS K. Lipid rafts as a membraneorganizing principle. Science 2010; 327: 46-50. https://doi. org/10.1126/science.1174621

[8] ZAJCHOWSKI LD, ROBBINS SM. Lipid rafts and little caves. Compartmentalized signalling in membrane microdomains. Eur J Biochem 2002; 269: 737-752.

[9] MOLLINEDO F, GAJATE C. Lipid rafts as major platforms for signaling regulation in cancer. Adv Biol Regul 2015; 57: 130-146. https://doi.org/10.1016/j.jbior.2014.10.003

[10] SIMONS K, TOOMRE D. Lipid rafts and signal transduction. Nat Rev Mol Cell Biol 2000; 1: 31-39. https://doi. org/10.1038/35036052

[11] HELMS JB, ZURZOLO C. Lipids as targeting signals: lipid rafts and intracellular trafficking. Traffic 2004; 5: 247-254. https://doi.org/10.1111/j.1600-0854.2004.0181.x

[12] ORLOWSKI S, MARTIN S, ESCARGUEIL A. P-glycoprotein and 'lipid rafts': some ambiguous mutual relationships (floating on them, building them or meeting them by chance?). Cell Mol Life Sci 2006; 63: 1038-1059. https://doi. org/10.1007/s00018-005-5554-9

[13] FENYVESI F, FENYVESI E, SZENTE L, GODA K, BACSO $\mathrm{Z}$ et al. P-glycoprotein inhibition by membrane cholesterol modulation. Eur J Pharm Sci 2008; 34: 236-242. https://doi. org/10.1016/j.ejps.2008.04.005
[14] LAVIE Y, FIUCCI G, LISCOVITCH M. Up-regulation of caveolae and caveolar constituents in multidrug-resistant cancer cells. J Biol Chem 1998; 273: 32380-32383. https:// doi.org/10.1074/jbc.273.49.32380

[15] SCHULTE T, PASCHKE KA, LAESSING U, LOTTSPEICH F, STUERMER CA. Reggie-1 and reggie-2, two cell surface proteins expressed by retinal ganglion cells during axon regeneration. Development 1997; 124: 577-587.

[16] Bickel PE, Scherer PE, Schnitzer JE, et al. Flotillin and epidermal surface antigen define a new family of caveolae-associated integral membrane proteins. J Biol Chem 1997; 272: 13793-13802. https://doi.org/10.1074/jbc.272.21.13793

[17] OTTO GP, NICHOLS BJ. The roles of flotillin microdomains--endocytosis and beyond. J Cell Sci 2011; 124: 39333940. https://doi.org/10.1242/jcs.092015

[18] BABUKE T, TIKKANEN R. Dissecting the molecular function of reggie/flotillin proteins. Eur J Cell Biol 2007; 86: 525532. https://doi.org/10.1016/j.ejcb.2007.03.003

[19] HANSEN CG, NICHOLS BJ. Molecular mechanisms of clathrin-independent endocytosis. J Cell Sci 2009; 122: 1713-1721. https://doi.org/10.1242/jcs.033951

[20] LANGHORST MF, SOLIS GP, HANNBECK S, PLATTNER $\mathrm{H}$, STUERMER CA. Linking membrane microdomains to the cytoskeleton: regulation of the lateral mobility of reggie-1/ flotillin-2 by interaction with actin. FEBS Lett 2007; 581: 4697-4703. https://doi.org/10.1016/j.febslet.2007.08.074

[21] GONZALO S, GRASA L, ARRUEBO MP, PLAZA MA, MURILLO MD. Extracellular signal-regulated kinase (ERK) is involved in LPS-induced disturbances in intestinal motility. Neurogastroenterol Motil 2011; 23: e80-90. https://doi. org/10.1111/j.1365-2982.2010.01632.x

[22] KIM EK, CHOI EJ. Compromised MAPK signaling in human diseases: an update. Arch Toxicol 2015; 89: 867-882. https://doi.org/10.1007/s00204-015-1472-2

[23] YAN X, RUAN W, WANG X, DING X, HAO L et al. [The effects of multidrug resistance on cell proliferation, apoptosis and invasion activity and the expression of mitogen-activated protein kinase in human hepatocellular cancer cells.] Zhong Liu 2012; 32: 507-515.

[24] LIAN WJ, LIU G, LIU YJ, ZHAO ZW, YI T et al. Downregulation of BMP6 enhances cell proliferation and chemoresistance via activation of the ERK signaling pathway in breast cancer. Oncol Rep 2013; 30: 193-200. https://doi. org/10.3892/or.2013.2462

[25] SHEN H, XU W, LUO W, ZHOU L, YONG W et al. Upregulation of mdr1 gene is related to activation of the MAPK/ ERK signal transduction pathway and YB-1 nuclear translocation in B-cell lymphoma. Exp Hematol 2011; 39: 558-569. https://doi.org/10.1016/j.exphem.2011.01.013

[26] BELLACOSA A, KUMAR CC, CRISTOFANO AD, TESTA JR. Activation of AKT Kinases in Cancer: Implications for Therapeutic Targeting. Adv Cancer Res 2005; 94: 29-86. https://doi.org/10.1016/S0065-230X(05)94002-5

[27] ISENOVIC ER, KEDEES MH, TEPAVCEVIC S, MILOSAVLJEVIC T, KORICANAC G et al. Role of PI3K/AKT, cPLA2 and ERK1/2 signaling pathways in insulin regulation of vascular smooth muscle cells proliferation. Cardiovasc Hematol Disord Drug Targets 2009; 9: 172-180. https://doi. org/10.2174/187152909789007034 
[28] HANADA M, FENG J, HEMMINGS BA. Structure, regulation and function of $\mathrm{PKB} / \mathrm{AKT}--\mathrm{a}$ major therapeutic target. Biochim Biophys Acta 2004; 1697: 3-16. https://doi. org/10.1016/j.bbapap.2003.11.009

[29] HENNESSY BT, SMITH DL, RAM PT, LU Y, MILLS GB. Exploiting the PI3K/AKT pathway for cancer drug discovery. Nat Rev Drug Discov 2005; 4: 988-1004. https://doi. org/10.1038/nrd1902

[30] STEELMAN LS, POHNERT SC, SHELTON JG, FRANKLIN RA, BERTRAND FE et al. JAK/STAT, Raf/MEK/ERK, $\mathrm{PI} 3 \mathrm{~K} / \mathrm{Akt}$ and BCR-ABL in cell cycle progression and leukemogenesis. Leukemia 2004; 18: 189-218. https://doi. org/10.1038/sj.leu.2403241

[31] CHANG F, LEE JT, NAVOLANIC PM, STEELMAN LS, SHELTON JG et al. Involvement of PI3K/Akt pathway in cell cycle progression, apoptosis, and neoplastic transformation: a target for cancer chemotherapy. Leukemia 2003; 17 : 590-603. https://doi.org/10.1038/sj.leu.2402824

[32] TESTA JR, BELLACOSA A. AKT plays a central role in tumorigenesis. Proc Natl Acad Sci U S A 2001; 98: 1098310985. https://doi.org/10.1073/pnas.211430998

[33] YANG C, HOU A, YU C, DAI L, WANG W et al. Kanglaite reverses multidrug resistance of HCC by inducing apoptosis and cell cycle arrest via PI3K/AKT pathway. Onco Targets Ther 2018; 11: 983-996. https://doi.org/10.2147/OTT. S153814
[34] WANG Y, QU Y, NIU XL, SUN WJ, ZHANG XL et al. Autocrine production of interleukin- 8 confers cisplatin and paclitaxel resistance in ovarian cancer cells. Cytokine 2011; 56: 365-375. https://doi.org/10.1016/j.cyto.2011.06.005

[35] LIN X, ZHANG X, WANG Q, LI J, ZHANG P et al. Perifosine downregulates MDR1 gene expression and reverses multidrug-resistant phenotype by inhibiting PI3K/Akt/ NF-kappaB signaling pathway in a human breast cancer cell line. Neoplasma 2012; 59: 248-256. https://doi.org/10.4149/ neo_2012_032

[36] KUO MT, LIU Z, WEI Y, LIN-LEE YC, TATEBE S et al. Induction of human MDR1 gene expression by 2 -acetylaminofluorene is mediated by effectors of the phosphoinositide 3-kinase pathway that activate NF-kappaB signaling. Oncogene 2002; 21: 1945-1954. https://doi.org/10.1038/ sj.onc. 1205117

[37] CHEN S, WANG Y, RUAN W, WANG X, PAN C. Reversing multidrug resistance in hepatocellular carcinoma cells by inhibiting extracellular signal-regulated kinase/mitogen-activated protein kinase signaling pathway activity. Oncol Lett 2014; 8: 2333-2339. https://doi.org/10.3892/ol.2014.2521 